Radial and Nonradial Pulsations as Probes of Stellar Physics

ASP Conference Series, Vol. 259, 2002

C. Aerts, T.R. Bedding, \& J. Christensen-Dalsgaard, eds.

\title{
Unusual Mode Changes in the $\delta$ Scuti Type Variable Star FL Cancri
}

\author{
V.P. Goranskij, A.V. Kusakin \\ Sternberg Astronomical Institute, Moscow University, Russia
}

\begin{abstract}
On the base of 105 hour photoelectric monitoring, we show that FL Cnc is a multiperiodic $\delta$ Sct type variable with rapid night-tonight changes of the amplitudes of the periodic components in the Fourier spectra. We observed an event of pulsating frequency doubling on a time scale of a week, which was treated as an azimuthal mode change.
\end{abstract}

The variability of FL Cnc (HD 74292 (A2), BD $+32^{\circ} 1782$, R.A. $=8^{h} 44^{m} 14^{s} .89$, Decl. $=+32^{\circ} 03^{\prime} 45^{\prime \prime} .1,2000.0$ ) was discovered by Kusakin (1994). The star has mean brightness $V=7^{m} .032$, an amplitude of variations in $V$ of ${ }^{m} .06$, and mean colors $B-V=0^{m} .233$ and $V-R=0^{m} .211$. It is suspected to be a member of the $\lambda$ Boo class (Mkrtichian, private communication). The first observations indicated that FL Cnc had a composite Fourier spectra changing radically from season to season. Kusakin \& Goranskij (1996) showed that the periodic components in its light curve changed more rapidly, on the time scale of a few days. Two frequencies, of 13 and $26 \mathrm{c} \mathrm{d}^{-1}$, having the largest amplitude components, were picked out. One of these frequencies dominated in the data of each separate season.

\section{Observations}

A total of 105.8 hours of monitoring in 19 nights of 1989 - 1994 were taken with 48-cm telescope of Tien-Shan Astronomical Observatory and photoelectric four-channel WBVR photometer. HD 75332 and HD 74057 were comparison and check stars. An extensive monitoring was performed in 6 nights between JD 2449365 and 2449371 to study rapid variations, the duration of each nightly series being 8-10 hours. Deeming (1975) frequency analysis, and decomposition of the light curve into periodic components, were applied to analyse the data.

\section{Results}

Each night, the power spectrum of a long, 8-10 hour series of observations has several periodic components, resembling other multiperiodic $\delta$ Sct type variable stars. The accuracy $\Delta f$ of a frequency $f$ revealed from a distinct night series of $\Delta T$ day long is represented by $\Delta \phi / \Delta T$, where $\Delta \phi$ is the uncertainty of the wave phase location which is equal to $0^{p} .1-0^{p} .5$ in our case. So, it is of $0.3-1.7 \mathrm{c}$ $\mathrm{d}^{-1}$. The frequencies measured in such a way are uniformly distributed over a wide range between 7 and $40 \mathrm{c} \mathrm{d}^{-1}$. The periodic components reach maximum amplitude when their frequencies are close to 13 or $26 \mathrm{c} \mathrm{d}^{-1}$. The amplitude 
- frequency diagram of the data points has a two-humped distribution, the hump of $13 \mathrm{c} \mathrm{d}^{-1}$ has about twice larger amplitude than that of $26 \mathrm{c} \mathrm{d}^{-1}$. The sample of peaks and peak amplitudes of components change from night to night. Some components disappear and others appear in the time scale of a few days. The light curve cannot be predicted with a simple multiperiodic model having constant amplitude components.

The monitoring carried out in six nearly consecutive nights shows the transit from the dominant oscillation with the period of $0^{d} .07461\left(f=13.4 \pm 0.3 \mathrm{c} \mathrm{d}^{-1}\right)$ to a more rapid one with a period of $0^{d} .03732\left(f=26.8 \pm 0.5 \mathrm{c} \mathrm{d}^{-1}\right)$, being exactly twice shorter (Fig. 1). In the power spectra, the process looks like continuous decay of the low frequency peak, and rise of the higher frequency one.

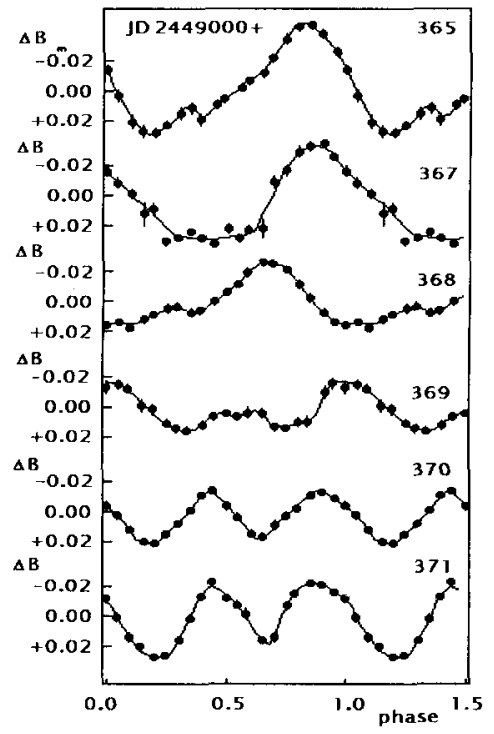

Figure 1. The nightly mean light curves of FL Cnc calculated with the linear elements

$$
T_{0}=2449365.000+0^{d} .07461 * E .
$$

The doubling of the pulsation frequency on the time scale of a week is seen.

\section{Conclusions}

Although FL Cnc may be classified as a multiperiodic $\delta$ Sct type star, its behaviour is unusual due to the rapid variability of the periodic components in its light curve from night to night.

We conclude that the dominating oscillations in FL Cnc with the frequencies of 13 and $26 \mathrm{c} \mathrm{d}^{-1}$ are probably azimuthal modes of order $|m|=1$ and $|m|=2$. Transitions between these modes occur. This effect is apparent in the amplitude - frequency diagram as a two maxima point distribution, the frequency ratio of these maxima being equal to $1: 2$.

\section{References}

Deeming, T.J. 1975, Ap\&SS, 36, 137

Kusakin, A.V. 1994, IBVS, No 4001

Kusakin, A.V.\& Goranskij, V.P. 1996, Astron. Reports, 40, 221 